\title{
Kyste osseux solitaire des maxillaires : caractéristiques radiologiques et données étiopathogéniques
}

\author{
Jean-Claude Harnet ${ }^{1, \star}$, Ahmed Féki ${ }^{2}$, Tommaso Lombardi ${ }^{3}$ \\ 1 Service de Pathologie et Chirurgie buccale, Hôpitaux universitaires, Nice, France \\ 2 Service de Pathologie et Chirurgie buccale, Hôpitaux universitaires, Strasbourg, France \\ 3 Laboratoire d'Histopathologie buccale et maxillo-faciale, division de Stomatologie et Chirurgie orale, Genève, Suisse
}

(Reçu le 6 janvier 2010, accepté le 14 janvier 2010)

Mots clés :

kyste osseux solitaire / radiographie / pathogénie

\begin{abstract}
Résumé - Le kyste osseux solitaire des maxillaires est constitué par une lésion ostéolytique, sans paroi épithéliale et au contenu variable selon le site osseux. Il réalise, dans sa localisation maxillaire, une cavité osseuse de forme géodique dont l'aspect radiologique est très souvent polymorphe. L'approche diagnostique de cette pseudo-tumeur osseuse bénigne ne repose en fait que sur la symptomatologie peu évocatrice, quelques caractéristiques sémiologiques en radiographie standard, la découverte peropératoire d'une cavité vide et l'analyse histologique qui montre l'absence de bordure épithéliale. Parfois, l'extension de la lésion lui donne un aspect très polymorphe et ouvre l'interprétation radiographique à un diagnostic différentiel très large.

Une synthèse des connaissances cliniques acquises à travers les localisations osseuses maxillomandibulaires et les localisations métaphysaires humérales et fémorales, beaucoup plus fréquentes, permet de discuter les principales hypothèses étiologiques émises concernant un processus pathogène qui semble varier selon les sites osseux affectés, le mode d'apparition, l'évolution et les réponses aux thérapeutiques médico-chirurgicales proposées.
\end{abstract}

\begin{abstract}
Solitary bone cyst of the jaws: radiographic features and pathogenesis. The solitary bone cyst of the jaws is an osteolytic lesion, devoid of an epithelial lining, with a content that varies according to the affected skeletal site. It appears as a unilocular cavity but often with a polymorphic radiological aspect. The diagnosis of this pseudo-cyst is mainly based on poor symptomatology, some radiological features on standard $\mathrm{X}$-ray examinations, and the intraoperative finding of an empty cavity along with the absence of an epithelial covering at microscopy. Occasionally, the extent of the lesion produces a polymorphic pattern, which gives rise to a wide array of differential diagnoses.

The aim of this paper is to summarize clinical knowledge in relation to both jaws as well as much more frequently observed femoral and humeral metaphyseal locations, and to discuss the main etiologic hypotheses based on presentation, evolution and therapeutic approaches which apparently vary according to the involved site.
\end{abstract}

Pour designer le kyste osseux solitaire (KOS), on a utilisé une terminologie très variée, parfois énigmatique (kyste idiopathique, kyste essentiel, kyste osseux simple) ou qui traduit le processus étiopathogénique suspecté (kyste osseux traumatique, hémorragique ou hématique, ostéodystrophie kystique). Cela démontre que le KOS des maxillaires reste encore une lésion très mal connue.

Dans notre spécialité, le KOS est classé parmi les bonerelated lesions dans les tumeurs odontogènes des maxillaires [1]. Il est défini par une lésion ostéolytique se

\footnotetext{
^Correspondance : jeanclaudeh7@gmail.com
}

traduisant par une image radiologique de forme géodique, parfois très polymorphe. Macroscopiquement, la lésion comporte une cavité osseuse, tapissée par une fine membrane conjonctive, la cavité peut être «vide » ou remplie par un liquide séreux, séro-hématique ou sanguin.

Le KOS manifeste une localisation préférentielle [2-5] pour les os longs (90\% des cas), surtout les régions métaphysaires de l'humérus $(65 \%)$ et du fémur $(25 \%)$, à proximité du cartilage de conjugaison. La lésion semble évoluer selon deux composantes : une extension centrifuge associée à une « migration » en direction diaphysaire, contemporaine de la croissance osseuse. La localisation maxillo-mandibulaire 


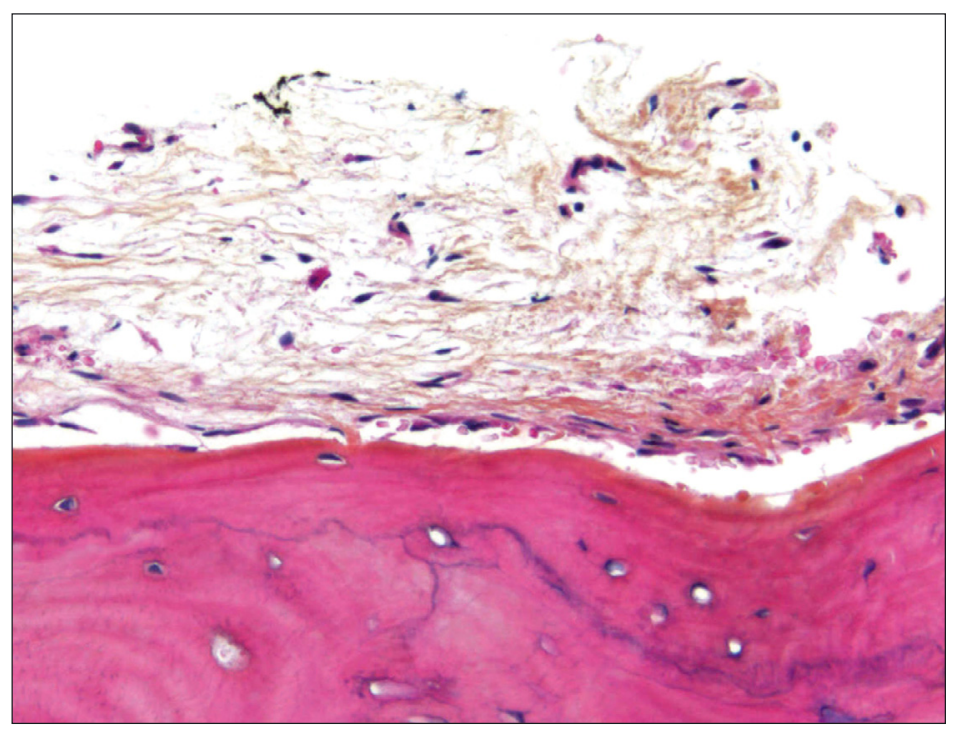

Fig. 1. Fragment osseux recouvert par une fine couche de tissu conjonctif fibreux (HES, $x$ 40).

Fig. 1. Bone fragment covered by a thin layer of fibrous connective tissue (HES, $x$ 40). est bien moins fréquente. En effet, dans les séries de lésions kystiques étudiées, la fréquence du KOS des maxillaires varie de 0,5 à $1,0 \%$ avec une atteinte préférentielle, généralement unilatérale, de la mandibule ( $75 \%$ des cas) et le siège de prédilection $[6,7]$ est constitué par la région antéromoyenne du corps de la mandibule avec des extensions lésionnelles postérieures parfois importantes. Dans ces deux localisations squelettiques, le diagnostic de KOS est établi au cours de l'enfance et de l'adolescence. La plupart des KOS sont découverts au cours des deux premières décennies, sans une prédominance marquée pour l'un des sexes pour la localisation maxillo-mandibulaire, mais avec une nette prédominance pour le sexe masculin pour la localisation extra-faciale.

Cliniquement, le KOS mandibulaire est le plus souvent asymptomatique, sans tuméfaction apparente, sans signes fonctionnels marqués : il est découvert fortuitement à la lecture d'un examen radiologique panoramique $[8,9]$. En revanche, l'histoire naturelle du KOS des os longs est marquée par une phase initiale asymptomatique, suivie plus ou moins rapidement d'une phase symptomatique caractérisée le plus souvent par une douleur vive qui signe une fracture pathologique spontanée; elle est observée dans $90 \%$ des cas dans la localisation humérale $[10,11]$.

L'examen macroscopique du KOS montre une paroi de kyste formée d'une membrane conjonctive mince, inconstante, d'aspect gris jaunâtre, friable et hémorragique. Cette paroi peut être également marquée par la présence de foyers de nécrose osseuse aseptique, beaucoup plus fréquents dans les localisations métaphysaires des os longs. Le contenu du kyste semble varier selon la durée d'évolution et la localisation de la lésion. Il peut s'agir d'un fluide sanguin, séro-hématique ou séreux. L'absence de contenu, donnant une cavité osseuse "vide», est particulièrement fréquente dans la localisation mandibulaire. Elle semble être liée essentiellement à la durée de la période d'évolution écoulée entre l'apparition de la lésion et son traitement. En effet, une période d'évolution relativement longue permettrait la résorption progressive [12] du fluide intrakystique alors que celui-ci serait présent lorsque la lésion est traitée très précocement.

En microscopie photonique, la paroi du kyste est formée d'une membrane de tissu conjonctif plus ou moins riche en fibres de collagène, totalement dépourvue d'épithélium de revêtement (Fig. 1). La paroi kystique ou plutôt le produit de curetage de la paroi osseuse comporte de nombreux fibroblastes, des cellules géantes ressemblant à des ostéoclastes sont parfois observées; on retrouve souvent quelques travées d'os néoformé dans l'épaisseur de la paroi, entourées d'un grand nombre d'ostéoblastes. Enfin de nombreux capillaires congestifs $[13,14]$ et des cristaux de cholestérol (en rapport avec les plages de nécrose osseuse aseptique) peuvent être également visibles.

\section{Sémiologie radiologique}

L'image radiologique standard du KOS mandibulaire varie essentiellement en fonction du degré d'extension de la lésion.

\section{Images radioclaires de faible étendue}

Dans cette configuration, le KOS peut se présenter sous la forme d'une image ostéolytique monogéodique, de forme conique, à apex vertical (Fig. 2) ou horizontal (Fig. 3). Le fond est homogène, limité par une bordure de condensation osseuse fine. Le diagnostic radiologique est rendu plus aisé par la reconnaissance d'une image radioclaire plus polymorphe, monogéodique ou polygéodique, avec un bord supérieur caractéristique, c'est-à-dire d'aspect ondulé, contournant les éléments radiculaires qui sont généralement respectés (Fig. 4). 


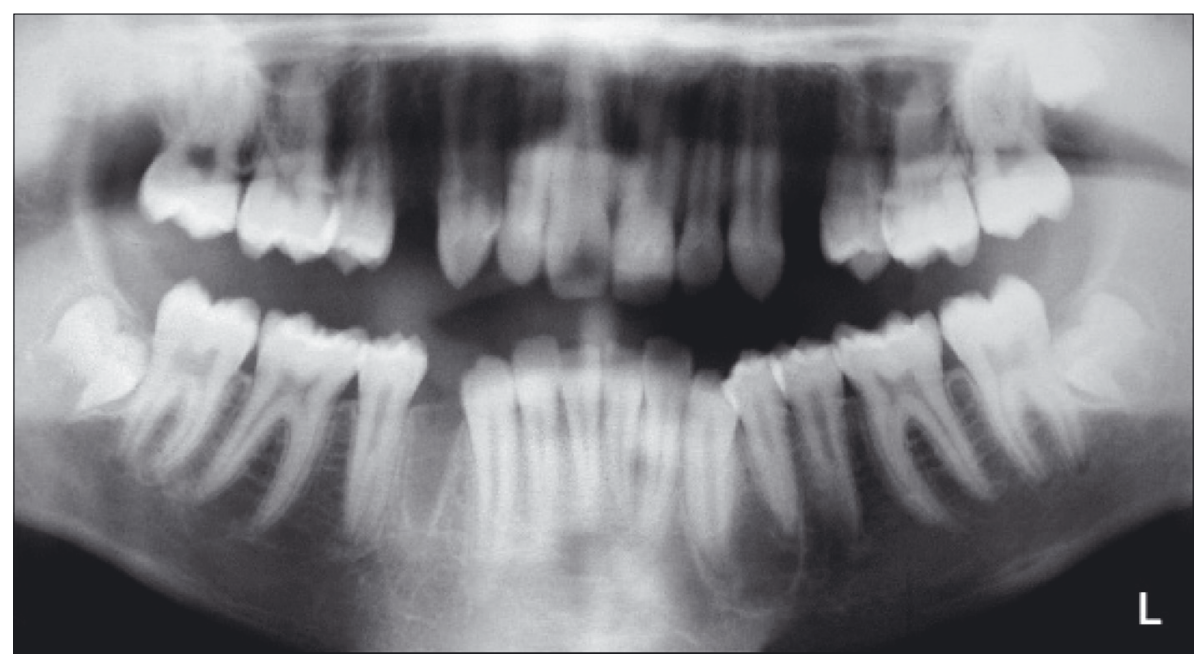

Fig. 2. KOS de l'hémi-mandibule gauche : image unigéodique (région 33-34), à apex vertical (garçon, 13 ans). Fig. 2. Solitary bone cyst of the left side of the mandible: unilocular, oblong image (region 33-34) (a 13 year-old boy).

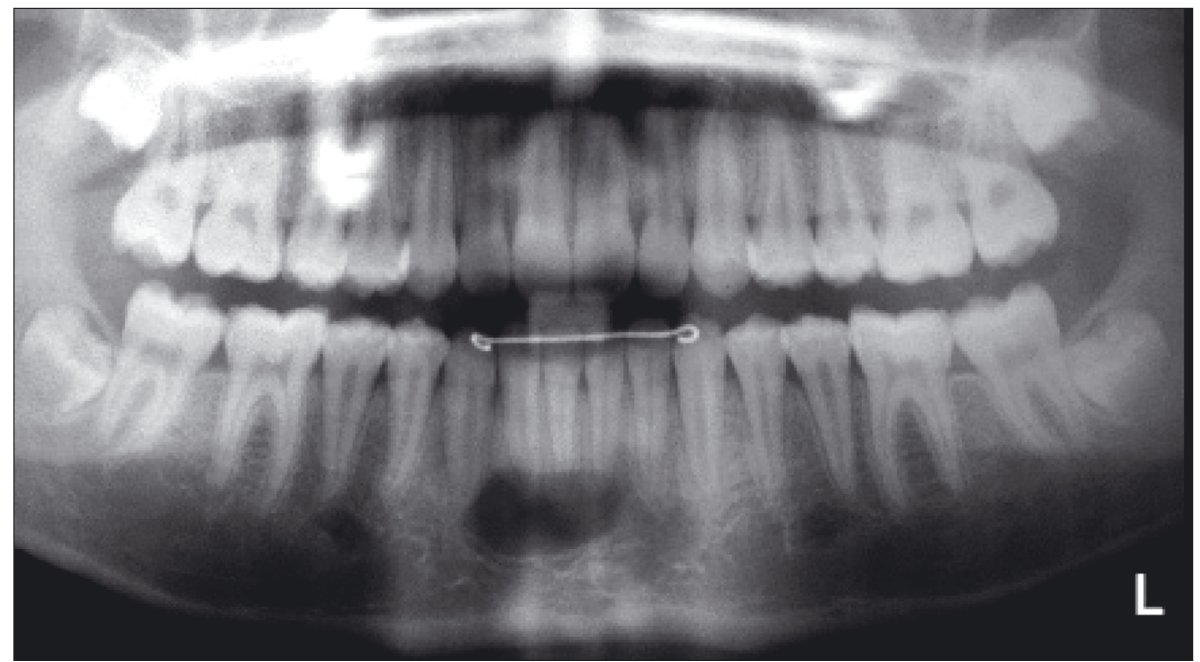

Fig. 3. KOS de la symphyse mandibulaire : image unigéodique, à apex horizontal (garçon, 14 ans). Fig. 3. Solitary bone cyst of the mandibular symphysis: oval unilocular image (a 14 year-old boy).

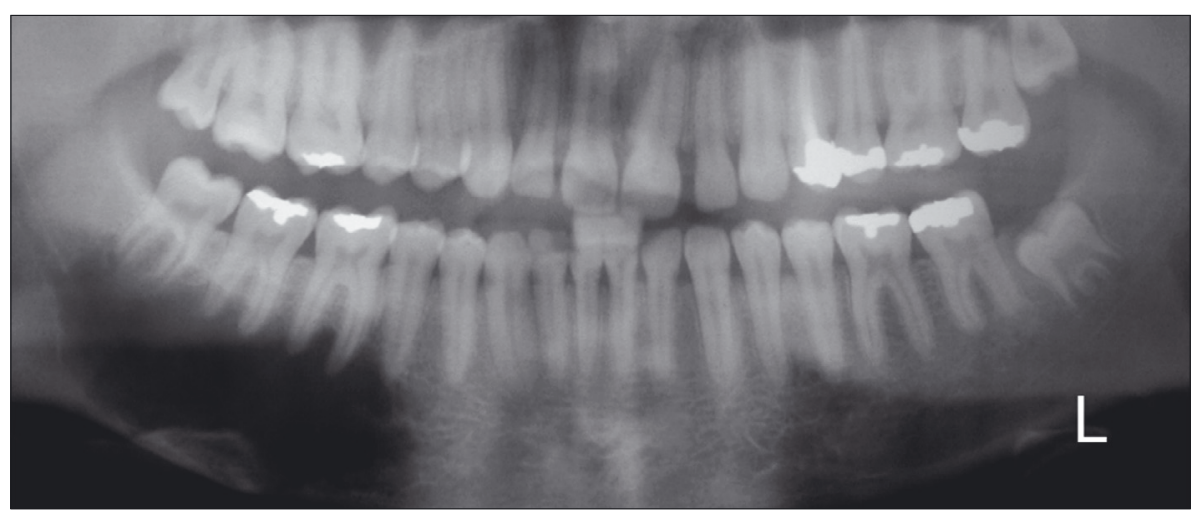

Fig. 4. KOS de l'hémi-mandibule droite : image ostéolytique avec bord supérieur ondulé (scalloping) de la lésion (garçon, 17 ans). Fig. 4. Solitary bone cyst of the right side of the mandible: osteolytic lesion with scalloped upper border (a 17 year-old boy). 


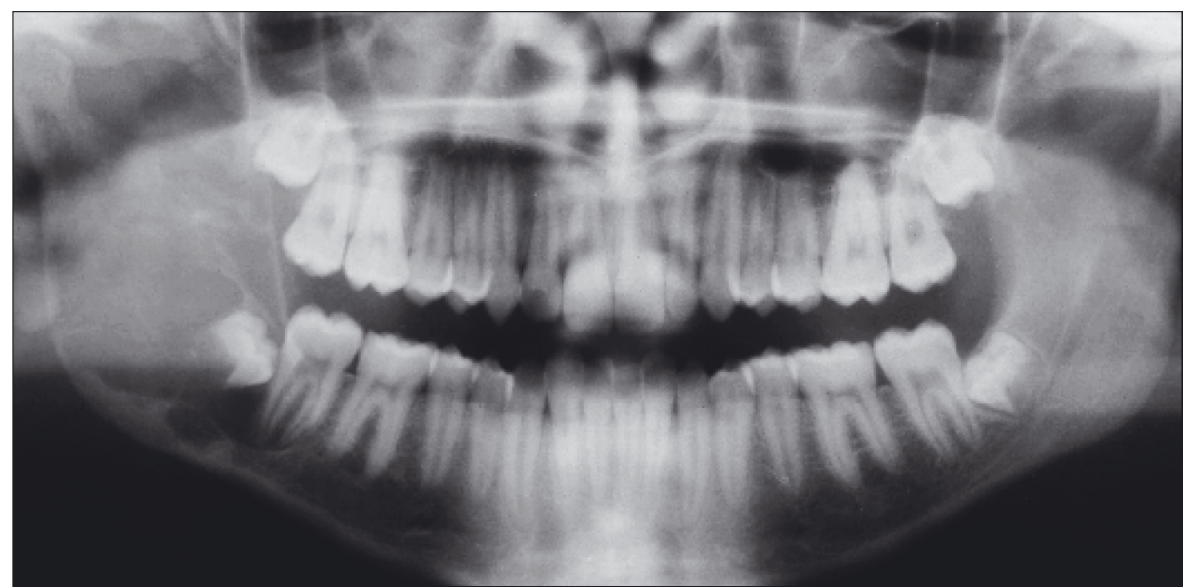

a

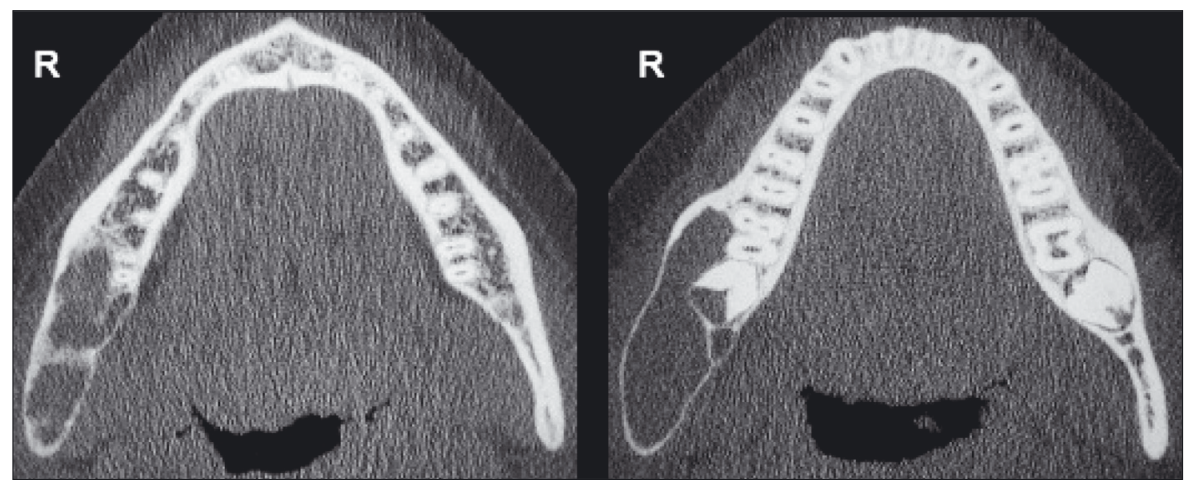

b

Fig. 5. a : Image ostéolytique polymorphe de l'angle et du ramus droits (fille, 16 ans). b : exploration TDM montrant le cloisonnement osseux. Fig. 5. a: Multilocular osteolytic lesion on the right angle and ramus of the mandible; (a 16 year-old girl). b: CT-scan exam showing internal septa.

\section{Images radioclaires de grande étendue}

À ce stade, l'image radiologique comporte un diagnostic différentiel plus large. En radiographie standard, elle peut être franchement polygéodique avec un fond d'aspect cloisonné (Fig. 5a) faisant évoquer des lésions tumorales de type kératokyste odontogène ou améloblastome. De telles images très extensives nécessitent une exploration tomodensitométrique qui montre, dans la quasi-totalité des cas de KOS, une corticale très amincie, soufflée, mais sans solution de continuité (Fig. 5b).

La densité de la lésion observée en scanographie est comparable à celle des autres kystes et tumeurs des maxillaires. Elle a fait l'objet de quelques interrogations en raison de la nature très différente des éléments constituant la paroi et la lumière de la cavité osseuse pathologique du KOS. L'apport de l'imagerie en résonance magnétique (IRM) est controversé : l'IRM avec amplification de contraste après injection de gadolinium autoriserait un diagnostic précis car elle permet de distinguer le KOS des autres lésions kystiques et tumorales $[15,16]$.

\section{Processus pathogène}

L'étiopathogénie du KOS, en particulier dans sa forme anatomoclinique maxillo-mandibulaire, est peu abordée dans la littérature. La confusion y est souvent entretenue par la difficulté de distinguer entre le processus pathogénique qui semble faire l'objet d'un consensus en orthopédie, et l'étiologie proprement dite qui est encore le sujet d'une controverse, surtout pour les facteurs déclenchants.

Bien que l'examen histologique en microscopie optique soit très souvent peu évocateur, on remarque classiquement l'absence des éléments caractéristiques d'un kyste et la présence de fragments d'os nécrosé; des zones de remodelage peuvent parfois être mises en évidence sur les parois de la cavité kystique. Cette nécrose osseuse, aseptique, permettrait d'expliquer l'origine vasculaire du KOS : il serait secondaire à une ischémie locale, induite par une hémorragie intramédullaire d'origine artérielle qui entraînerait une altération du drainage veineux réalisé par le réseau intramédullaire $[17,18]$. Cette hypothèse semble corroborée par la fréquence élevée des KOS observées chez les sujets atteints de drépanocytose; cette maladie génétique, à transmission 
autosomique récessive, qui affecte la synthèse de l'hémoglobine, se traduit par des crises douloureuses dues à des microinfarctus, y compris dans l'os, secondaires à des phénomènes vaso-occlusifs [19]. L'extension du KOS serait aussi favorisée par l'augmentation de la pression intramédullaire [20], due à l'accumulation du fluide intrakystique qui a une composition proche du sérum sanguin. Il contient donc des éléments enzymatiques provenant des zones de remodelage osseux présentes sur la paroi du KOS où la vascularisation est très dense. Les analyses biologiques montrent que le fluide kystique correspond à un transsudat ne contenant aucun microorganisme [21], mais avec des facteurs enzymatiques à fortes concentrations [22]. Ces facteurs, comme les métalloprotéinases, responsables de l'activité ostéoclasique de la lésion, pourraient être impliqués à la fois dans les phénomènes d'ostéogenèse et d'ostéoclasie [23].

\section{Hypothèses pathogéniques}

L'étiologie proprement dite du KOS, quelle que soit sa localisation squelettique, n'est pas encore parfaitement élucidée et de nombreuses théories peuvent être retrouvées dans la littérature. Trois d'entre elles font l'objet d'une discussion récurrente impliquant une anomalie dans les phénomènes de croissance osseuse, un processus évolutif de dégénérescence tumorale, ou un facteur déclenchant « traumato-hémorragique ».

\section{Anomalies de la croissance osseuse}

Le KOS se développe généralement pendant la préadolescence ou l'adolescence, dans une zone de remodelage osseux très actif ou à son voisinage. Cette constatation a fait suspecter une relation entre le développement du KOS et le remodelage osseux. La terminologie ancienne d'« ostéodystrophie kystique », utilisée par Mikulicz (1906), Rushton (1946) et Jaffe (1953) pour désigner le KOS, traduisait l'hypothèse étiopathogénique d'une anomalie dans le développement et la croissance du tissu osseux.

Une anomalie locale de la croissance pourrait expliquer les localisations préférentielles du KOS au voisinage des cartilages de conjugaison [24-26] qui semblent constituer une barrière à l'expansion kystique vers l'épiphyse mais aussi un facteur favorisant l'activité et l'extension kystique vers la diaphyse. Pendant la croissance, les points d'ossification métaphysaires des os longs constituent des zones de remodelage osseux très actif et, pour certains auteurs, le KOS se développerait à partir de zones de remodelage « hors contrôle » [27].

À la mandibule, le point d'ossification primaire siège à proximité immédiate du foramen mentonnier, région anatomique de prédilection du KOS. On peut donc aussi suspecter une anomalie de la différenciation cellulaire au cours des phénomènes très complexes d'ossification et de croissance. Cette anomalie pourrait entre autres être favorisée par des facteurs environnementaux locaux qui exerceraient des contraintes mécaniques sur l'ostéogenèse et l'angiogenèse [28].

Toutefois, la diversité et la complexité des mécanismes impliqués dans l'ossification et dans la croissance mandibulaire ne permettent pas de formuler une hypothèse aussi explicite que pour les os longs. De plus, l'ossification mandibulaire est d'origine mixte, à la fois membraneuse et enchondrale.

\section{Dégénérescence tumorale}

Plusieurs hypothèses évoquent une transformation tumorale survenant au cours du développement de lésions ostéodystrophiques. Par exemple, un KOS pourrait apparaître à partir d'une lésion dysplasique fibreuse par dégénérescence du tissu fibreux et formation de microkystes [29]. Cette hypothèse a été évoquée en raison de la localisation préférentielle de la dysplasie fibreuse pour la diaphyse et les maxillaires.

Autre hypothèse, le KOS pourrait résulter de la « liquéfaction » centrale d'un granulome centro-osseux à cellules géantes $[18,30]$; la liquéfaction constituerait une étape du processus de guérison. Là encore, c'est la localisation préférentielle du granulome central à cellules géantes dans les régions épiphysaire et symphysaire qui a fait évoquer cette hypothèse. Enfin, certaines formes de dysplasie ostéo-cémentaire pourraient participer au développement d'un KOS [6].

\section{Traumatisme}

L'étiopathogénie «traumato-hémorragique » constitue l'hypothèse la plus souvent avancée. Cette théorie s'appuie sur l'apparition d'une hémorragie intramédullaire suivie d'un hématome après un traumatisme insuffisant pour provoquer la fracture d'un os sain. La pression exercée par l'hématome entraînerait une stase veineuse favorisant la nécrose de la moelle osseuse $[2,20,31]$, la résorption ostéoclasique serait stimulée par la diminution du pH tissulaire $[32,33]$.

Le traumatisme pourrait être également à l'origine d'une thrombose ou d'un spasme durable de l'artère terminale, entraînant une ischémie et une nécrose aseptique déterminant la formation du KOS. Des anomalies de la vascularisation sont probablement à l'origine des phénomènes de résorption, les conditions qui mènent à la différentiation des ostéoclastes font encore l'objet de discussion.

Le rôle du traumatisme reste très controversé : les données épidémiologiques montrent que plus de $50 \%$ des cas de KOS n'ont pas d'antécédents traumatiques et que les localisations préférentielles du KOS ne correspondent pas strictement aux zones les plus exposées; cela est vrai en particulier pour certaines localisations extra-faciales. Cette théorie étiologique pourrait être compatible avec l'apparition des lésions à la mandibule qui est souvent le siège, par ailleurs, de microtraumatismes iatrogènes. 


\section{Conclusion}

Le KOS n'est pas une lésion spécifique des maxillaires. Une synthèse des connaissances cliniques acquises à travers les localisations osseuses maxillo-mandibulaires et celles, beaucoup plus fréquentes, qui concernent les métaphyses humérales et fémorales, a permis de discuter les principales hypothèses étiopathogéniques émises. Celles-ci tentent $d$ 'expliquer un processus pathologique qui varie, en fonction des sites osseux affectés, par son mode d'apparition, son évolution et la réponse au traitement chirurgical. De ces différentes hypothèses, il ressort néanmoins un certain « consensus » concernant la pathogénie ostéolytique qui résulterait d'une altération du système vasculaire, réalisant une ischémie locale post-hémorragique à l'origine d'une véritable nécrose osseuse aseptique et de l'apparition d'un transsudat dans la cavité kystique, contenant de nombreux facteurs enzymatiques responsables de la résorption osseuse. La composante étiologique proprement dite, induisant la genèse tumorale, met en cause traumatismes, dégénérescence tumorale ou anomalies liées à la croissance osseuse, processus étiologiques le plus souvent retenus mais toujours controversés.

Le KOS des maxillaires et le KOS retrouvé dans les autres localisations squelettiques sont des lésions qui présentent quelques similitudes dans leur aspect clinique et leur étiopathogénie. Le KOS des maxillaires a une évolution favorable après un traitement simple tandis que le KOS des os longs peut comporter des séquelles très invalidantes, ce qui fait discuter pour certains sa nature bénigne.

\section{Références}

1. Barnes L, Eveson JW, Reichart P, Sidransky D. WHO classification of tumours. Pathology \& genetics. In: Head and neck tumours. Odontogenic tumours. IARC Press, Lyon, 2005.

2. Ottolenghi CE, Schajowicz F, Raffa J. Le kyste osseux essentiel uniloculaire. Étude clinique et anatomopathologique de 123 cas. Rev Chir Orthop 1969;55:287-303.

3. Normann A, Schiffman M. Simple bone cysts: factors of age dependency. Radiology 1977;124:779-82.

4. Filho RJG, Dos Santos JBG, Korukian M, Filho JL. Conservative treatment of solitary bone cysts: a study of 55 patients. Rev Paul Med 1992;10:131-37.

5. Bensahel $H$, Jehanno P, Desgrippes $Y$, Pennecot GF. Solitary bone cyst: controversies and treatment. J Pediatr Ortho Part B 1998;7:257-61.

6. Kaugars GE, Cale AE. Traumatic bone cyst. Oral Surg Oral Med Oral Pathol 1987;63:318-24.

7. Howe GL. Haemorrhagic cysts of mandible. Br J Oral Surg 1965;3:55-91.

8. Adachi K, Shigehara H, Ochi S, Nakatsu T, Akita K, Kishi K. Clinico-radiographic evaluation of simple bone cyst accompanied by radiopaque lesions. Oral Radiol 1993;9:27-34.

9. Ludwig A. Merten HA. Kystes osseux traumatiques : pathogénèse, clinique et thérapie. Rev Mens Suisse Odontostomatol 1995;105:66-70.

10. Kaelin AJ, Mc Even GD. Unicameral bone cysts. Natural history and the risk of fracture. Int Orthop 1989;13:275-82.
11. Kaelin AJ. Kyste osseux solitaire de l'enfant (pp. 135-52). Sauramps, Montpellier, 1996.

12. Xanthinaki AA, Choupis KI, Tosios K, Pagkalos VA, Papanikolaou SI. Traumatic bone cyst of the mandible of possible iatrogenic origin: a case report and brief review of the literature. Head Face Med 2006;2:40.

13. Jaffe HL, Lichtenstein L. Solitary unicameral bone cyst. Arch Surg 1942;44:1004-25.

14. Schajowicz F. Solitary bone cyst (simple or unicameral bone cyst) (p. 417-24). Tumors and tumor-like lesions of bone and joints. Springer, Berlin-Heidelberg-New York, 1981.

15. Suomalainen A, Apajalahti S, Kuhlefelt M, Hagstrom J. Simple bone cyst: a radiological dilemma. Dentomaxillofac Radiol 2009;38:174-7.

16. Matsuzaki H, Asaumi JI, Yanagi $Y$, Konouchi H, Honda $Y$, Hisatomi M, Shigehara H, Kishi K. MR Imaging in the assessment of solitary bone cyst. Eur J Radiol Extra 2003;45:37-42.

17. Rubin MM, Murphy FJ. Simple bone cyst of mandibular condyle. J Oral Maxillofac Surg 1989;47:1096-98.

18. Kuboyama K, Shido T, Harada A, Yokoe S. Therapy of solitary unicameral bone cyst with percutaneous trepanation. Rinsho Seikei Geka 1981;16:288-93.

19. Noujaim S, Alantar A, Galeazzi JM, Genet P, Maman L. Kyste osseux solitaire chez un patient atteint d'une drépanocytose. Med Buccale Chir Buccale 2003;9:105-11.

20. Chigira M, Maehara S, Arita S, Udagawa E. The aetiology and treatment of simple bone cysts. J Bone Joint Surg $\mathrm{Br}$ 1983;65:633-37.

21. Harris SJ, Carroll K0, Gordy FM. Idiopathic bone cavity (traumatic bone cyst) with the radiographic appearance of a fibroosseous lesion. Oral Surg Oral Med Oral Pathol 1992;74:118-23.

22. Kaelin AJ. Kyste osseux solitaire de l'enfant (p. 135-52). Sauramps, Montpellier, 1996.

23. Komiya S, Inoue A. Development of a solitary bone cyst - a report of a case suggesting its pathogenesis. Arch Orthop Trauma Surg 2000;120:455-7.

24. Bradley J.C. Solitary bone cyst of the zygomatic bone. Br Dent J 1982, 152: 203-04.

25. Cudia G, Gordeef A, Panzoni E. Le kyste osseux solitaire : une observation à double localisation mandibulaire et humérale. Rev Stomatol Chir Maxillofac 1987;88:31-5.

26. Ludwig A, Merten HA. Kystes osseux traumatiques : pathogenèse, clinique et thérapie des kystes osseux traumatiques. Rev Mens Suisse Odontostomatol 1995;105:66-70.

27. Broder HM, Chester W. Possible precursor of unicameral bone cysts. J Bone Joint Surg 1968;50A:503-7.

28. Lefranc J, Nezelof C. Les kystes essentiels des os longs. Étude anatomo-pathologique et traitement chirurgical. Rev Chir Orthop 1957;43:385-406.

29. Hara H, Ohishi M, Higuchi Y. Fibrous dysplasia of the mandible associated with large solitary bone cyst. J Oral Maxillofac Surg 1990;48:88-91.

30. Abril JC, Queiruga JA, Casas J, Albinana J. Unusual finding after contrast injection of a solitary bone cyst. A case report. Acta Orthop Belg 1999;65:235-8.

31. Mayer R, Libotte M, Ruppol P. La lacune essentielle de la mandibule. Acta Stomatol Belg 1967;64:33-52.

32. Howe GL. Haemorrhagic cysts of the mandible - I. Br J Oral Surg 1965;3:55-76.

33. Howe GL. Haemorrhagic cysts of the mandible - II. Br J Oral Surg 1965;3:77-91. 